\title{
MAGNETIC FLARES ON ASYMPTOTIC GIANT BRANCH STARS
}

\author{
Noam Soker ${ }^{1,2}$ and Joel H. Kastner ${ }^{1}$
}

\begin{abstract}
We investigate the consequences of magnetic flares on the surface of asymptotic giant branch (AGB) and similar stars. In contrast to the solar wind, in the winds of AGB stars the gas cooling time is much shorter than the outflow time. As a result, we predict that energetic flaring should enhance, rather than inhibit, dust formation around AGB stars. Analysis of an archival ROSAT X-ray spectrum of the Mira system yields results for intrinsic X-ray luminosity $\left(\sim 2 \times 10^{29}\right.$ erg $\left.\mathrm{s}^{-1}\right)$ and temperature $\left(\sim 10^{7} \mathrm{~K}\right)$ that are consistent with coronal emission from the AGB star Mira A, although it also possible that the X-ray emission arises from accretion onto its hot companion, Mira B.
\end{abstract}

Subject headings: stars: mass loss — stars: winds, outflows - X-rays: ISM stars: AGB — stars: magnetic fields

\section{INTRODUCTION}

Asymptotic giant branch (AGB) stars represent the final stages of evolution for intermediate mass $\left(\sim 1-8 M_{\odot}\right)$ stars, just before these stars (potentially) generate planetary nebulae. Such stars have exhausted their core hydrogen, have completed core helium and $\mathrm{H}$-shell burning phases, and are now burning $\mathrm{H}$ and He in concentric shells around the exhausted stellar core (this core, once exposed, will become a degenerate white dwarf). AGB stars are ascending fully convective Hayashi tracks in the H-R diagram, and the very large luminosities of these stars $\left(\sim 10^{4} L_{\odot}\right)$, in combination with copious dust grain formation in their cool, extended atmospheres, lead to dusty, radiatively-driven winds. Hence, AGB stars lose mass rapidly, with rates ranging from $10^{-8}$ to $10^{-4} M_{\odot} \mathrm{yr}^{-1}$ (as ascertained from far-IR continuum and millimeter-wave molecular line emission; e.g. Loup et al. 1993).

\footnotetext{
${ }^{1}$ Chester F. Carlson Center for Imaging Science, Rochester Institute of Technology, 54 Lomb Memorial Dr., Rochester, NY 14623; jhk@cis.rit.edu

${ }^{2}$ Department of Physics, Oranim, Tivon 36006, ISRAEL; soker@physics.technion.ac.il
} 
There are several independent pieces of evidence for the presence of magnetic fields in and around cool giants and, in particular, in and around AGB stars. These include:

1. Maser polarization around AGB stars and other cool stars. SiO masers are found close to (typically within less than a few stellar radii of) the stellar surface (e.g. Kemball \& Diamond 1997), OH masers are found at at $\sim 10^{15}-10^{16} \mathrm{~cm}$ from the star (e.g., Szymczak, Cohen, \& Richards 1999; Palen \& Fix 2000), and $\mathrm{H}_{2} \mathrm{O}$ masers are found in between (Vlemmings, Diamond, \& van Langevelde 2002).

2. Polarization of $\mathrm{OH}$ maser spots in planetary nebulae $(\mathrm{PNs})$ and proto-PNs, which are the immediate descendants of AGB stars (e.g., Zijlstra et al. 1989). Miranda et al. (2001) find polarization in the $1,665-\mathrm{MHz} \mathrm{OH}$ maser line, which indicates the presence of $\sim 10^{-3} \mathrm{G}$ magnetic fields at $\sim 10^{16} \mathrm{~cm}$ from the central star of the young PN K3-35.

3. X-ray emission from cool giant stars (Hünsch et al. 1998; Schröder, Hünsch, \& Schmitt 1998; Hünsch 2001). Hünsch (2001) used Chandra to detect X-ray emission from two $\mathrm{M}$ giant stars, which display hard X-ray luminosities of $L_{x} \simeq 10^{30} \mathrm{erg} \mathrm{s}^{-1}$.

Although it is widely agreed that radiation pressure on dust is the main acceleration process of winds from AGB stars (Elitzur, Ivezic, \& Vinkovic 2002, and references therein), there is still disagreement over the role the magnetic field plays in shaping the circumstellar matter. First, it is not clear whether magnetic fields play any role in shaping the winds; many other models exist, e.g., acceleration via radiation pressure on dust in fast rotating AGB stars spun up by companions (e.g., Dorfi \& Höfner 1996; Reimers, Dorfi \& Höfner 2000), and the influence of a companion outside the envelope (e.g., Mastrodemos \& Morris, M. 1999; Soker 2001). Second, there is a disagreement on whether the magnetic fields play a dynamical role, i.e., whether the magnetic pressure and/or tension become comparable to the other relevant forces in the flow (e.g., Pascoli 1997; Chevalier \& Luo 1994; García-Segura 1997; García-Segura et al. 1999; García-Segura, \& López 2000; García-Segura, López, \& Franco 2001; Matt et al. 2000; Blackman et al. 2001; Gardiner \& Frank 2001; Falceta-Goncalves \& Jatenco-Pereira 2002; see review by García-Segura 2002), or whether the magnetic field is relatively weak on a global scale, and plays only secondary effects (Soker 2002a; Soker \& Zoabi 2002 and references therein).

In earlier papers Soker and collaborators (e.g., Soker 1998; Soker \& Clayton 1999) propose that cool magnetic spots facilitate the formation of dust, hence locally increasing the mass loss rate. In this process the magnetic field has a secondary role and becomes dynamically important only in relatively small regions of the stellar surface and circumstellar environment. In this model the large scale magnetic field is too weak to play a dynamic role 
and directly influence the wind from the AGB star. However, the magnetic field is strong enough above and near cool spots that reconnection can occur; such reconnection events likely would lead to magnetic flares analogous to those on the surface of the sun. The reconnection process is basically cancellation of magnetic field lines having opposite directions. The energy released by the reconnecting magnetic fields heats the gas and accelerates it to high speeds. The gas is heated by MHD shocks (e.g., Chen et al. 1999). In the present paper we discuss some implications of these events. In $\S 2$ the potential of impact of flaring on circumstellar dust formation is considered. In $\S 3$ we discuss the expected observational signatures of AGB star magnetic activity in general and of flares in particular, and we analyze the X-ray spectrum of a prototypical AGB star, Mira, to explore the possibility that magnetic activity may lead to X-ray emission from AGB stars. Our summary is in $\S 4$.

\section{DUST FORMATION IN FLARES}

Whereas Soker (1998) and Soker \& Clayton (1999) argued that magnetic starspots on cool giants can lead to enhanced dust formation, we demonstrate here that reconnection events (flares) associated with such spots may themselves further enhance the formation rate of dust. Because the density close to the surface of AGB stars is very high, the question regarding dust formation reduces to the value of the temperature. To evaluate the temperature resulting from a flare, the time scale of cooling should be compared with the flow time. In the sun the conduction time is non-negligible compared with the radiative cooling time (e.g., Aschwanden et al. 2000). However, in AGB stars it can be neglected (this can be shown by using equations 16 and 17 from Aschwanden et al. 2000).

Let us consider an idealized simple case, in which the magnetic field expands radially from the stellar surface outward, while the density is determined by an isothermal hydrostatic halo. In such a case, the magnetic pressure decreases relatively slowly, $B(r)=B_{*}\left(r / R_{*}\right)^{-2}$, where $B_{*}$ is the magnetic pressure on the stellar surface, and $R_{*}$ is the stellar radius (subscript * will stand for quantities on the stellar surface). For an isothermal gas above and close to the photosphere the density is given by

$$
\rho=\rho_{*} \exp \left[-\left(r-R_{*}\right) / H_{*}\right]
$$

where the scale height close to the stellar surface is

$$
H \equiv R_{*} \frac{C_{*}^{2}}{v_{K}^{2}} \simeq 0.045 R_{*}\left(\frac{T_{*}}{3000 \mathrm{~K}}\right)\left(\frac{R_{*}}{1 \mathrm{AU}}\right)\left(\frac{M_{*}}{M_{\odot}}\right)^{-1} .
$$

In equation (2), $C_{*}$ and $v_{K}$ are the isothermal sound speed and Keplerian velocity on the stellar surface, respectively. For reconnection to occur and heat the gas substantially, the 
magnetic pressure should overwhelm the thermal pressure. This occurs several scale heights above the surface, and higher. In the case of the sun the same condition is met very close to the solar surface, and we can have small flares as well as large flares. The smaller flares (nanoflares) above the solar surface have minimum height of $h \sim 0.0015 R_{\odot} \simeq 4 H_{\odot}$ (e.g., Aschwanden et al. 2000). Inside magnetic flux loops the magnetic pressure is high, and pressure equilibrium with the loop exterior implies that the density inside the loop is smaller than in its surroundings. Also, after heating following reconnection, the hot gas expands and the density drops below the simple isothermal halo value. Strong flares extend to much larger distances from the solar surface, $h \simeq 0.007-0.07 R_{\odot} \simeq 20-200 H_{\odot}$ (e.g., Aschwanden et al. 2000).

As can be seen from equation (2), on the surface of AGB stars, for which $R_{*} \gtrsim 1 \mathrm{AU}$, the scale height is $H \sim 0.05 R_{*}$. As just noted for the case of the sun, flares, from small to large, extend from $h \sim 4 H$ to $h \sim 200 H$ above the solar surface. Taking the same range for AGB stars, for which $H \simeq 0.05 R_{*}$ (Eq. 2), we find that flares will occur over the range of $r=R_{*}+h \simeq 1.2-10 R_{*}$ Since in the upper range $h \gg R_{*}$, it is not clear if flares can extend to such large radii above the surfaces of AGB stars.

The typical density of the photospheres of AGB stars is $n_{e}\left(R_{*}\right) \sim 10^{14} \mathrm{~cm}^{-3}$ (Soker \& Harpaz $\left.1999^{3}\right)$. In the simple isothermal halo used above, the density drops to $n_{e} \simeq 10^{10} \mathrm{~cm}^{-3}$ at $r \sim 1.3 R_{*}$. At distances of $r \gtrsim 2 R_{*}$ we are in the region where density is determined by the wind, i.e., $\rho=\dot{M} /\left(4 \pi r^{2} v_{w}\right)$, where $\dot{M}$ is the mass loss rate into the wind (defined positively), and $v_{w}(r)$ is the wind speed. For a fully ionized wind - which is not the case for the global AGB wind, but will be the case locally after a flare event - the scaled electron density is

$$
n_{e} \simeq 10^{10}\left(\frac{\dot{M}}{10^{-6} M_{\odot} \mathrm{yr}^{-1}}\right)\left(\frac{r}{1 \mathrm{AU}}\right)^{-2}\left(\frac{v_{w}}{10 \mathrm{~km} \mathrm{~s}^{-1}}\right)^{-1} \mathrm{~cm}^{-3}
$$

where, for simplicity, we have assumed isotropic mass loss. Using the cooling function from, e.g., Gaetz, Edgar \& Chevalier (1988), we find the isobaric radiative cooling time in the temperature range $2 \times 10^{5} \mathrm{~K} \lesssim T \lesssim 4 \times 10^{7} \mathrm{~K}$ to be

$$
\tau_{\text {rad }} \simeq 800\left(\frac{n_{e}}{10^{10}}\right)^{-1}\left(\frac{T}{10^{6}}\right)^{3 / 2} \mathrm{~s} .
$$

The flow time across the reconnection region can be estimated as follows. Let the size of the reconnection flux tube be $x \simeq H \sim 10^{12} \mathrm{~cm}$, and the flow speed be the sound speed

\footnotetext{
${ }^{3}$ Note that the density scale in Figs. 1-5 of Soker \& Harpaz 1999 is too low by a factor of 10; the correct scale is displayed in their Fig. 6.
} 
at $T=10^{6} \mathrm{~K}$, i.e., $v_{\exp } \sim 100 \mathrm{~km} \mathrm{~s}^{-1}$. The expansion time is then

$$
\tau_{\exp } \sim 10^{5}\left(\frac{x}{H}\right)\left(\frac{H}{10^{12} \mathrm{~cm}}\right)\left(\frac{v_{e}}{100 \mathrm{~km} \mathrm{~s}^{-1}}\right)^{-1} \mathrm{~s} .
$$

Even if $x \sim 0.1 H$, the radiative cooling time of gas heated by flares to $T \sim 10^{6} \mathrm{~K}$ in AGB stars is much shorter than the expansion time. The cooling time will also be shorter than the expansion time in strong and large flares, having a temperature of $T \sim 10^{7} \mathrm{~K}$ and a size of $x \gtrsim 10 H$. Close to the AGB stellar surface the wind does not yet reach its terminal velocity, hence the density is higher and the cooling time shorter than the values in equations (3) and (4), respectively. In the sun, where $H_{*} \sim 3 \times 10^{7} \mathrm{~cm}$, we find that the situation is different, with the radiative cooling time much longer than the expansion time (see, e.g., Aschwanden et al. 2000). At larger distances from the surface of AGB stars, $r \sim 10 R_{*}$, the cooling time is no longer much shorter than the expansion time; nevertheless, the condition that the cooling time is much shorter than the expansion time holds for dust formation close to the stellar surface.

The compression in the strong shock that results from the reconnection event can be larger than a factor of 4 . This is because the gas is not fully ionized. For conditions above cool giants, and for a shock temperature of $\sim 10^{6} \mathrm{~K}$, the post shock density can be a factor $\sim 5$ - 10 larger than the pre-shock density (Woitke, Gores \& Sedlmayr 1996a). Since the gas cools much faster than expansion time, to maintain pressure equilibrium the gas will be further compressed. The situation here is more complicated than the shocks generated by pulsating stars, as discussed by Woitke et al. (1996a), because the shocks are MHD shocks (e.g., Chen et al. 1999). After the gas cools from $T \sim 10^{6}$ to $T \sim 10^{4} \mathrm{~K}$, we can take, therefore, a density that is larger by a factor of $\sim 10$ relative to the value in equation $(3)$.

After this gas cools to $T \simeq 10^{4} \mathrm{~K}$, the radiative cooling time increases substantially (e.g., Woitke, Krüger, \& Sedlmayr 1996b); on the other hand, the cooling now proceeds radiatively as well as via adiabatic expansion (Woitke et al. 1996a). To form dust, the temperature should decrease to $T \lesssim 10^{3} \mathrm{~K}$. Using figure (11) of Woitke et al. (1996b), we approximate the cooling time from a temperature of $T \simeq 10^{4} \mathrm{~K}$ down to dust-forming temperatures by

$$
\tau_{\text {rad }} \simeq 5 \times 10^{6}\left(\frac{n_{e}}{10^{10} \mathrm{~cm}^{-3}}\right)^{-0.3} \mathrm{~s} .
$$

The cooler gas will expand at slower velocity; either the sound speed or the wind speed give $v_{e} \simeq 10 \mathrm{~km} \mathrm{~s}^{-1}$, which gives for the expansion time $\tau_{e} \sim 10^{6} \mathrm{~s}$, according to equation (5). Taking $n_{e} \sim 10^{11} \mathrm{~cm}^{-3}$, as discussed above, and considering further cooling by adiabatic expansion (Woitke et al. 1996a), we find the expansion time of the gas to be comparable to 
the cooling time for the scaling used above, i.e., a mass loss rate of $\dot{M}=10^{-6} M_{\odot} \mathrm{yr}^{-1}$ in equation (3).

The main conclusion from this discussion is that magnetic flares on the surfaces of AGB stars, as well as on other classes of actively mass-losing cool giant stars, can enhance dust formation in the range $\sim 1.2 R_{*} \lesssim r \lesssim 3 R_{*}$, as long as the mass loss rate is $\dot{M} \gtrsim$ $10^{-6} M_{\odot} \mathrm{yr}^{-1}$. The condition may be somewhat different for very massive cool giants, which we do not consider here, although the same qualitative mechanism may apply.

\section{OBSERVATIONAL SIGNATURES OF AGB STAR MAGNETIC ACTIVITY}

As the preceding suggests, it is likely that AGB stars generate locally strong surface magnetic fields, resulting in violent reconnection events (flares). Establishing the presence of such transient magnetic activity on AGB stars observationally would have several important ramifications; in particular, we would gain new insights into stellar dynamos and the shaping of planetary nebulae. We now discuss several new observational directions that show promise for establishing the degree of magnetic activity around AGB stars.

\subsection{X-ray emission and flaring}

Magnetic activity - due either to differential rotation in convective regions or, for very young systems, to interactions between magnetic fields associated with star and circumstellar disk - is widely believed to be ultimately responsible for X-ray emission from late-type stars. The physical conditions that give rise to this activity, and the connection between, e.g., stellar magnetic dynamo activity and X-ray emission, remains uncertain. It is apparent, however, that highly convective late-type stars and young, low-mass stars that presumably retain high rotation velocities and/or accretion disks are among the strongest X-ray emission sources. Most low-mass, pre-main sequence ( $\mathrm{T}$ Tauri) stars, for example, have X-ray luminosities (relative to bolometric) exceeding that of the Sun by 3-4 orders of magnitude (Feigelson \& Montmerle 1999). Hence, if AGB stars indeed generate strong magnetic fields, either locally or globally, then we expect them to be sources of X-ray emission and, perhaps, strong, long-duration (see below) X-ray flares.

The AGB phase is short-lived, such that there are relatively few nearby $(D<200 \mathrm{pc})$ examples, and (as already noted) AGB stars are generally enshrouded in expanding envelopes of X-ray-absorbing gas and dust. It is therefore not surprising that no unambiguous X-ray 
detections of AGB stars have been reported as of yet and that, in particular, no AGB stars were detected in the ROSAT All-Sky Survey. The only potential X-ray detection of an AGB star thus far, to our knowledge, is that of a prototypical object, Mira (o Ceti). At a distance of $128 \mathrm{pc}$, Mira is one of the closest AGB stars to Earth. ROSAT detected Xray emission toward Mira during a 9144 s pointed Position-Sensitive Proportional Counter (PSPC) observation on 1993 July 15 (Karovska et al. 1996). The PSPC count rate was 0.007 $\mathrm{s}^{-1}$. Karovska et al. (1996) attribute this X-ray emission not to coronal emission from Mira, but to accretion onto Mira's hot companion. Other observations also suggest the existence of an accretion disk (e.g., Bochanski \& Sion 2001; Wood, Karovska \& Raymond 2002).

We have used the PSPC spectrum ${ }^{4}$, albeit very noisy, to constrain the physical conditions of the X-ray emitting region under the assumption that the emission originates in solar-like coronal activity at the AGB star, Mira A. Specifically, we attempted fits of an absorbed Raymond-Smith plasma model to the PSPC spectrum (Fig. 1). We find the best-fit X-ray emission temperature and absorbing column are $T_{x}=1.1 \times 10^{7} \mathrm{~K}$ and $N_{H}=8.6 \times 10^{20}$ $\mathrm{cm}^{-2}$ (with formal uncertainties of $\sim \pm 10 \%$ ), with a total (absorbed) model flux of $6.1 \times 10^{-14}$ ergs $\mathrm{cm}^{-2} \mathrm{~s}^{-1}(0.2-2.2 \mathrm{keV})$. The inferred absorbing column is consistent with the modest mass-loss rate of Mira $\left(\sim 10^{-7} M_{\odot} \mathrm{yr}^{-1}\right)$ as deduced from circumstellar CO radio line emission (e.g., Loup et al. 1993), and is in rough agreement with the H I column density deduced from ultraviolet observations of $\mathrm{H}_{2}$ fluorescence (Wood et al. 2002).

The corresponding intrinsic (unabsorbed) X-ray luminosity is $L_{x}=2 \times 10^{29} \mathrm{erg} \mathrm{s}^{-1}$, such that $L_{x} / L_{b o l}$ is of order $10^{-7}$ if the emission indeed arises from the AGB star. This result for unabsorbed $L_{x}$ is in the range expected for the modest field strengths predicted by some models of magnetic activity on AGB stars (Soker \& Zoabi 2002). Thus, it is conceivable that Mira itself is an example of a magnetically active AGB star. However, we cannot rule out the possibility that the emission arises from accretion onto Mira B (Karovska et al. 1996; Wood et al. 2002 note that it is not clear yet if Mira B is a white dwarf or a main sequence star). Spectroscopy with Newton-XMM, which is superior to ROSAT in terms of both its effective area and spectral resolution, may help distinguish between the alternative emission mechanisms (i.e., coronal activity vs. accretion onto the hot companion), although the exceptional spatial resolution of the Chandra X-ray Observatory likely provides the only means to determine unambiguously the source of the X-ray emission. The separation between Mira A and B, $\sim 0.6^{\prime \prime}$ (Karovska et al. 1997), is just at the limit of Chandra's resolving power.

\footnotetext{
${ }^{4}$ These ROSAT data were obtained through the the High Energy Astrophysics Science Archive Research Center, a service of the Laboratory for High Energy Astrophysics at NASA/GSFC and the High Energy Astrophysics Division of the Smithsonian Astrophysical Observatory.
} 
In $\S 2$ we found that magnetic flares close to the the surfaces of AGB stars, and other cool giant stars, have scales of $\gtrsim 0.2 R_{*} \sim 5 \times 10^{12} \mathrm{~cm}$. If magnetic field reconnection proceeds at the sound speed of the gas heated to $\gtrsim 10^{6} \mathrm{~K}$, which is some fraction of the Alfven speed prior to reconnection, then the duration of a typical flare is $\tau_{\text {flare }} \gtrsim 5$ days. A long flare might last for of order a year. This is in contrast to flares on solar-like stars, which have typical time scales of $1-10^{4} \mathrm{~s}$ (e.g., Pettersen 1989). Another source of flares, consisting of transient, bright emission from the optical through the X-ray regimes, can be accretion events onto close companions, including brown dwarfs and massive planets (Struck, Cohanim \& Willson 2002). (The latter mechanism likely can't account for the X-ray properties of the Mira system, however, as X-ray emission generated via accretion onto a low-mass companion would be softer than that observed.) As with magnetic flares on main sequence stars and brown dwarfs, the typical flare timescales of such events should be very short compared with those expected from magnetic flares on AGB stars. It is clear, therefore, that long-duration

X-ray observations of AGB stars are very desirable as a means of establishing the presence and timescales of flares and, hence, distinguishing between potential flare sources.

We also concluded in the last section that for high enough mass loss rate, typically $\dot{M} \gtrsim 10^{-6} M_{\odot} \mathrm{yr}^{-1}$, flares can enhance dust formation; in Mira the mass loss rate is too low for that. The magnetic fields can also enhance dust formation via the formation of cool spots (e.g., Soker 1998; Soker \& Clayton 1999). Once local dust formation occurs, further dust formation can occur because of shadowing of the stellar radiation (Woitke, Sedlmayr, \& Lopez 2000), hence further increasing the inhomogeneity of the wind. Thus, in addition to searching for evidence for flaring via X-ray observations of AGB stars, it would be desirable to conduct coordinated X-ray and infrared observing campaigns designed to detect enhanced dust formation episodes during flares.

\subsection{Polarized radio-line maser emission: magnetic clouds in AGB winds?}

Estimates of magnetic field intensities from polarization in maser spots around AGB stars give high values (e.g., Vlemmings et al. 2002). Although the exact estimated values of the magnetic field are uncertain (Elitzur 1996), the estimates are still accurate to an order of magnitude (Elitzur, M., private communication, 2002), and in many maser spots implies that the magnetic pressure is much larger than the thermal pressure (Vlemmings et al. 2002). In the solar wind, on average, the magnetic pressure does not exceed the thermal pressure. However, the magnetic pressure does exceed the thermal pressure in magnetic clouds (e.g., Burlaga 2001; Yurchyshyn et al. 2001). Magnetic clouds are formed by impulsive mass loss events from the sun, and they are characterized by stronger than average magnetic fields, 
low proton temperatures, and smooth rotation of the magnetic field direction (e.g., Burlaga 2001). We suggest that the maser spots with strong magnetic fields which are observed around some AGB stars are similar in nature to the magnetic clouds in the solar wind, in that they represent local enhancements of the magnetic field (Soker 2002a; see also Dorch \& Freytag 2002 for a possible local dynamo in Betelgeuse). On average, we expect the magnetic pressure in the wind of AGB stars to be much below the thermal pressure, as winds from AGB stars are driven by radiation pressure on dust, rather than magnetic activity as in the sun.

\section{SUMMARY}

Recently, evidence has accumulated for the presence of relatively strong magnetic fields around AGB and similar stars. We have investigated certain theoretical and observational consequences of magnetic activity in such cool giant stars. The main results of this investigation are as follows.

1. Magnetic reconnection events near the stellar surface should lead to localized, long duration (timescales $\sim$ few days to a year) flares.

2. In contrast to the solar wind, in the expanding circumstellar envelopes of AGB stars the post-flare gas cooling time is much shorter than the typical flow timescale. As a result, we predict that energetic flaring should enhance, rather than inhibit, dust formation around AGB stars.

3. To explore the possible observational manifestations of AGB magnetic activity, we analyzed an archival ROSAT X-ray spectrum of the prototypical long-period variable star Mira. We find that the X-ray luminosity and temperature of the Mira system are consistent with coronal emission from the AGB star, Mira A, although it also possible that the X-ray emission arises from accretion onto the hot companion, Mira B.

4. Observations of polarized maser emission from the inner circumstellar envelopes of AGB stars may indicate the presence of localized, highly magnetized wind clumps analogous to magnetic clouds in the solar wind - rather than large-magnitude global magnetic fields.

We acknowledge support for this research provided by NASA/CXO grants GO0-1067X and GO2-3009X to RIT. N.S. acknowledges support from the US-Israel Binational Science Foundation and the Israel Science Foundation. 


\section{REFERENCES}

Aschwanden, M. J., Tarbell, T. D., Nightingale, R. W., Schrijver, C. J., Title, A., Kankelborg, C. C., Martens, P., \& Warren, H. P. 2000, ApJ, 535, 1047

Blackman, E. G., Frank, A., Markiel, J. A., Thomas, J. H., \& Van Horn, H. M. 2001, Nature, 409,485

Bochanski, J. J., \& Sion, E. M. 2001, AAS, 198, 1208

Burlaga, L. F. 2001, P\&SS, 49, 1619

Chen, P. F., Fang, C., Tang, Y. H., \& Ding, M. D. 1999, ApJ, 513, 516

Chevalier, R. A., \& Luo, D. 1994, ApJ, 421, 225

Dorch, S. B. F., \& Freytag, B. 2002, in Modeling of Stellar Atmospheres, IAU Sym., eds., N. E. Piskunov, W. W. Weiss, and D. F. Gray, (astro-ph/0208523)

Dorfi, E. A., \& Höfner, S. 1996, A\&A, 313, 605

Elitzur, M. 1996, ApJ, 457, 415

Elitzur, M., Ivezic, Z., \& Vinkovic, D. 2002, in Workshop on Mass-Losing Pulsating Stars and their Circumstellar Matter, in press (astro-ph/0207513)

Falceta-Goncalves, D., \& Jatenco-Pereira , V. 2002, ApJ, 576, 976

Feigelson, E. D., \& Montmerle, T. 1999, ARAA, 37, 363

Gaetz, T. J., Edgar, R. J., \& Chevalier, R. A. 1988, ApJ, 329, 927

García-Segura, G. 1997, ApJ, 489, L189

García-Segura, G. 2002, in Planetary Nebulae and their Role in the Universe, IAU Sym. 209, ASP Conference Series, in press (astro-ph/0202041)

García-Segura, G., Langer, N., Rozyczka, M., \& Franco, J. 1999, ApJ, 517, 767

García-Segura, G., \& López, J. A. 2000, ApJ, 544, 336

García-Segura, G., López, J. A., \& Franco, J. 2001, ApJ, 560, 928

Gardiner, T. A., \& Frank, A 2001, ApJ, 557, 250

Hünsch, M. 2001, in Astronomical Gesellschaft Abstract Ser., Vol. 18, MS 0710

Hünsch, M., Schmitt, J. H. M. M., Schröder, K.-P., \& Zickgraf, F.-J. 1998, A\&A, 330, 225

Karovska, M., Raymond, J., \& Guinan, E. 1996, Technical Report, Smithsonian Astrophysical Observatory

Karovska, M., Hack, W., Raymond, R., \& Guinan, E. 1997, ApJ, 482, L175 
Loup, C., Forveille, T., Omont, A., \& Paul, J. F. 1993, A\&AS, 99, 291

Kemball, A. J., \& Diamond, P. J., 1997, ApJ, 481, L111

Mastrodemos, N., \& Morris, M. 1999, ApJ, 523, 357

Matt, S., Balick, B., Winglee, R., \& Goodson, A. 2000, ApJ, 545, 965

Miranda, L. F., Gomez, Y., Anglada, G., \& Torrelles, J. M. 2001, Nature, 414, 284

Palen, S., \& Fix, J. D. 2000, ApJ, 531, 391

Pascoli, G. 1997, ApJ, 489, 946

Pettersen, B. R. 1989, SoPh, 121, 299

Reimers, C., Dorfi, E. A., \& Höfner, S. 2000, A\&A, 354, 373

Schröder, K.-P., Hünsch, M., \& Schmitt, J. H. M. M. 1998, A\&A, 335, 591

Soker, N. 1998, MNRAS, 299, 1242

Soker, N. 2001, ApJ, 558, 157

Soker, N. 2002a, MNRAS, in press, (astro-ph/0204157)

Soker, N., \& Clayton, G. C. 1999, MNRAS, 307, 993

Soker, N., \& Harpaz, A. 1999, MNRAS, 310, 1158

Soker, N., \& Zoabi, E. 2002, MNRAS, 329, 204

Struck, C., Cohanim, B. E., \& Willson, L. A. 2002, ApJ, 572, L83

Szymczak, M., Cohen, R. J., \& Richards, A. M. S. 1999, MNRAS, 304, 877

Vlemmings, W. H. T., Diamond, P. J., \& van Langevelde H. J. 2002, Proceeding of WS on Mass-Losing Pulsating Stars and their Circumstellar Matter, Y. Nakada \& M. Honma (eds), Kluwer ASSL series, in press (astro-ph/0208052)

Woitke, P., Goeres, A, \& Sedlmayr, E. 1996a, A\&A, 313, 217

Woitke, P., Krüger, D., \& Sedlmayr, E. 1996b, A\&A, 311, 927

Woitke, P., Sedlmayr, E., \& Lopez, B. 2000, A\&A, 358, 665

Wood, B. E., Karovska, M., \& Raymond, J. C. 2002, ApJ, 575, 1057

Yurchyshyn, V. B., Wang, H., Goode, P. R., \& Deng, Y. 2001, ApJ, 563, 381

Zijlstra, A. A., te Lintel Hekkert, P., Pottasch, S. R., Caswell, J. L., Ratag, M., \& Habing, H. J. 1989, A\&A, 217, 157

This preprint was prepared with the AAS IATEX macros v5.0. 


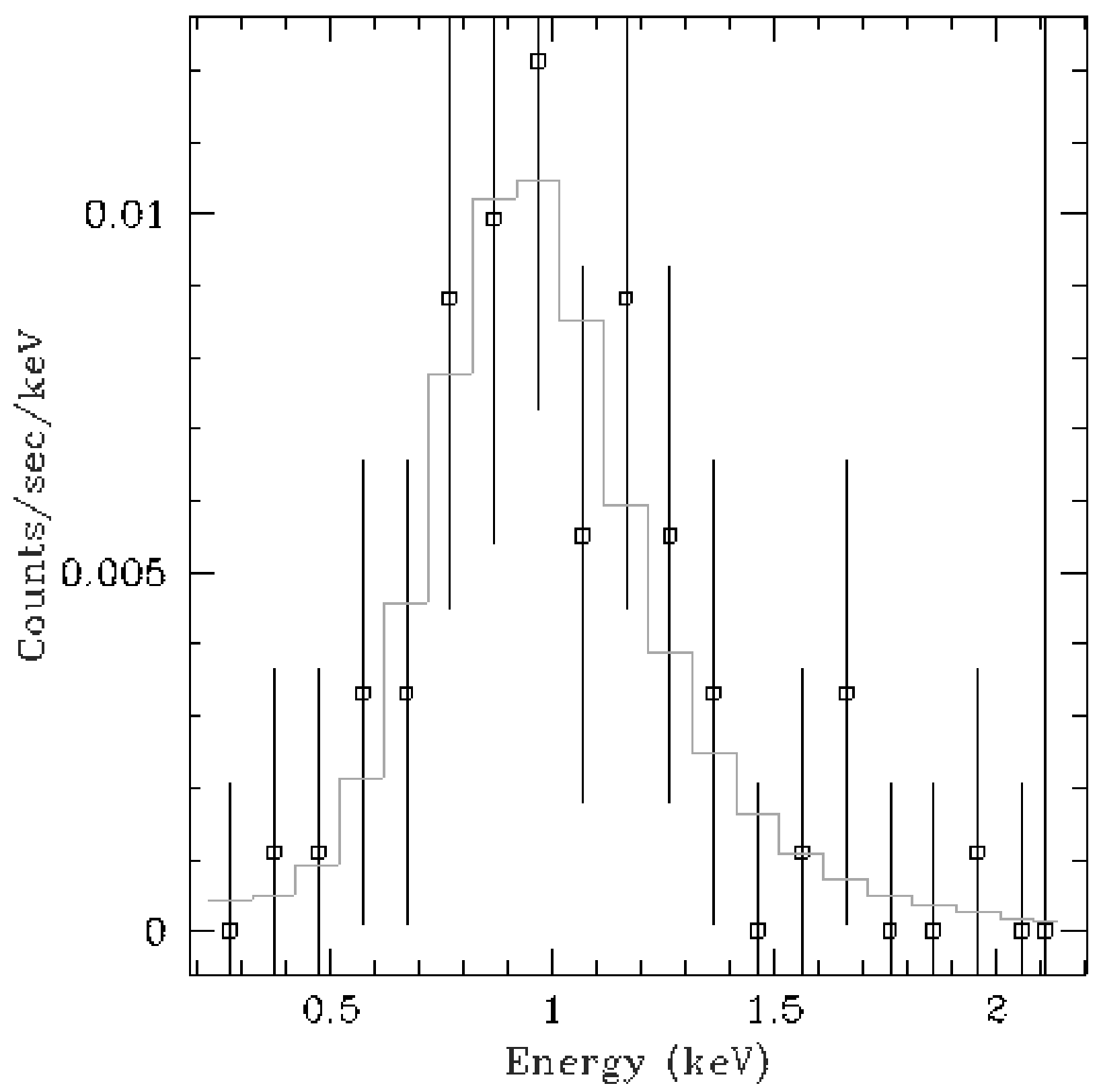

Fig. 1. - Archival ROSAT PSPC spectrum of the Mira system (squares), overlaid with best-fit absorbed Raymond-Smith plasma model (histogram). 